\title{
Hierarchical active poly(vinylidene fluoride) membrane fabricated by in-situ generated zero-valent iron for fouling reduction
}

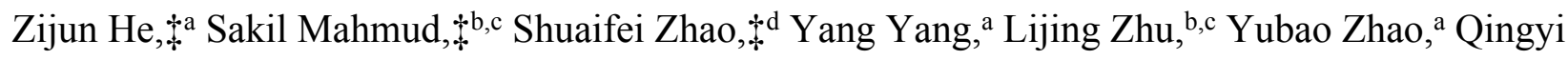
Zeng, ${ }^{\text {a }}$ Zhu Xiong, ${ }^{* a}$ and Chun $\mathrm{Hu}^{\mathrm{a}}$

a: Institute of Environmental Research at Greater Bay; Key Laboratory for Water Quality and Conservation of the Pearl River Delta, Ministry of Education, Guangzhou University, Guangzhou, Guangdong 510006, PR China.

b: Ningbo Institute of Materials Technology and Engineering, Chinese Academy of Sciences, Ningbo, Zhejiang 315201, PR China.

c: University of Chinese Academy of Sciences, Beijing 100049, PR China.

d: Deakin University, Geelong, Institute for Frontier Materials, VIC 3216, Australia.

* Corresponding author: Tel: +86-10-39346609; Fax: +86-10-62923541

E-mail: xiongzhu@gzhu.edu.cn; 


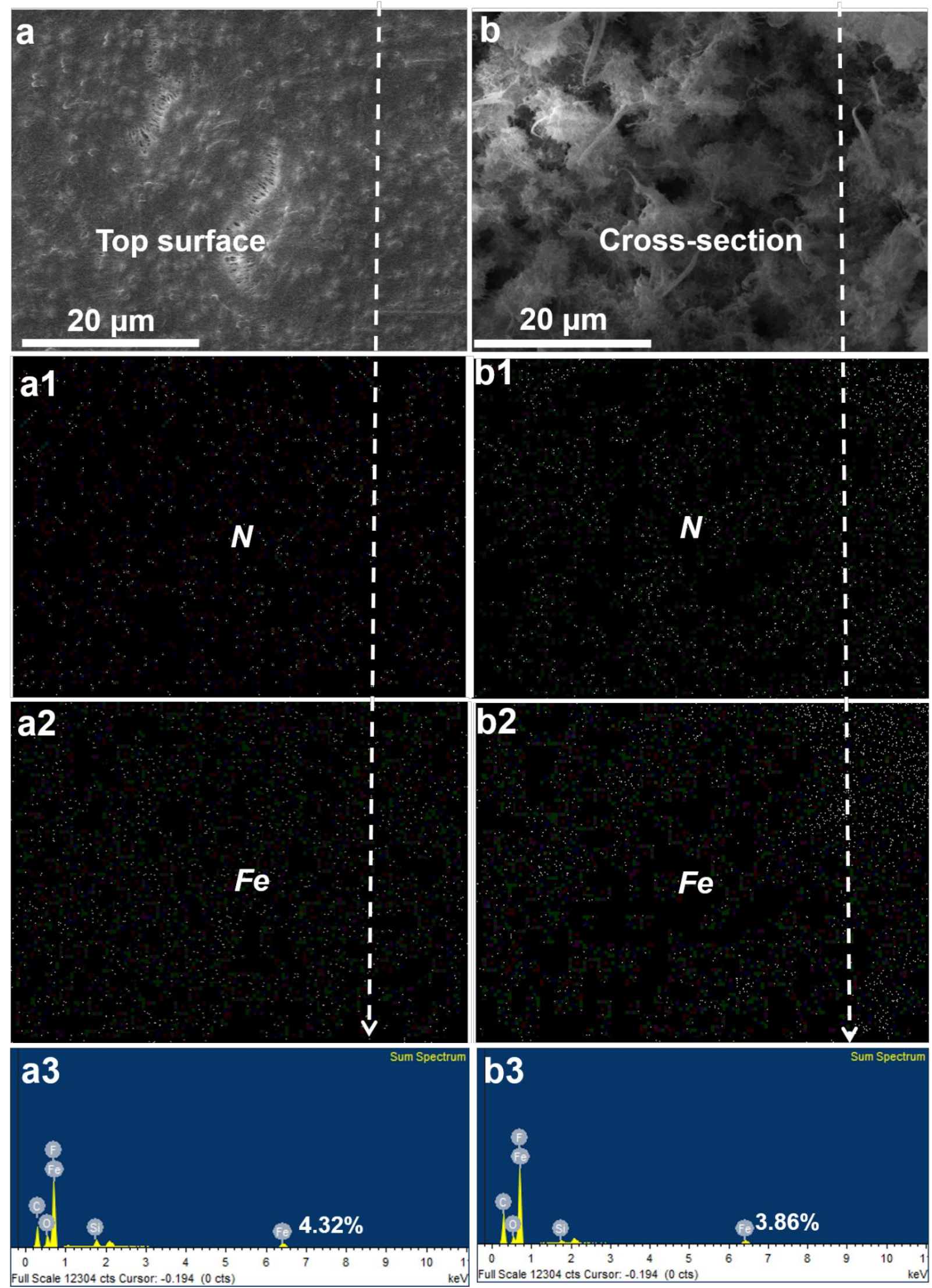

Figure S1 ( $a, b)$ SEM images of the top surface and cross-section of Fe NPs-loaded PVDF 
membrane. (a1, a2) N and Fe maps of EDX from (a), (b1, b2) N and Fe maps of EDX from (b). (a3)

EDS spectra from a. (b3) EDS spectra from b.

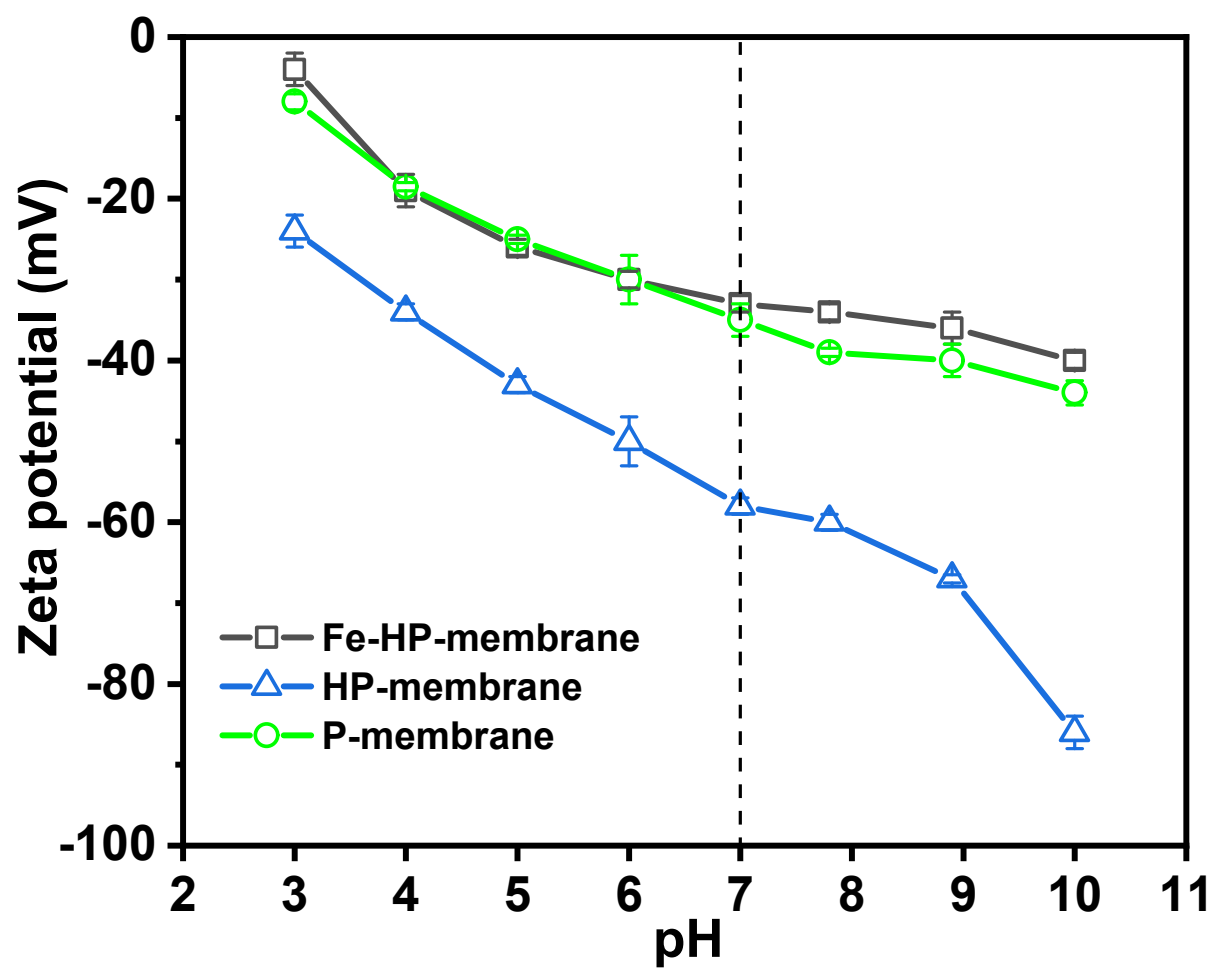

Figure S2 The zeta potential of P-membrane, HP-membrane and Fe-HP-membrane.
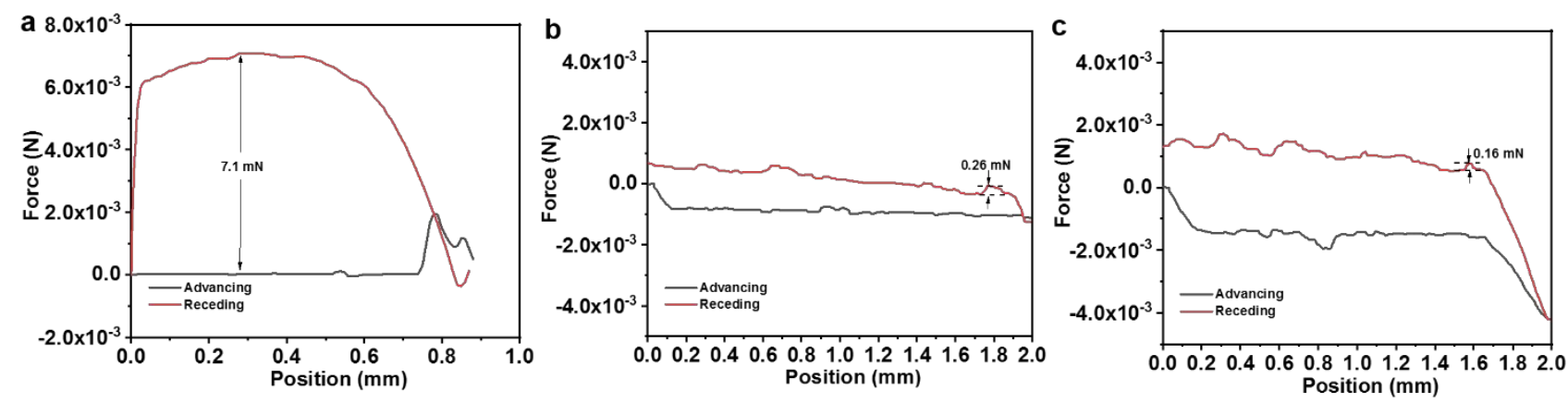

Figure S3. The surface affinity of (a) P-membrane, (b) HP-membrane and (c) Fe-HP-membrane to oil during the dynamic adhesion measurements.

The adhesion forces of oil under water on the PVDF membrane samples were measured using a highsensitive microelectromechanical balance system (Data-Physics DCAT11, Germany). 
Table S1 Chemical/physical cleaning for humic acid fouling of composite membranes.

\begin{tabular}{|c|c|c|c|c|c|}
\hline $\begin{array}{l}\text { Base material and } \\
\text { reference }\end{array}$ & $\begin{array}{l}\text { Feeding } \\
\text { solution }\end{array}$ & $\begin{array}{l}\text { Chemical/p } \\
\text { hysical } \\
\text { cleaning }\end{array}$ & Cleaning condition & Initial flux & Final flux recovery \\
\hline $\begin{array}{l}\text { Active PVDF UF } \\
\text { (This work) }\end{array}$ & $\begin{array}{l}\text { Humic acid } \\
(1000 \mathrm{ppm})\end{array}$ & $\begin{array}{l}\mathrm{H}_{2} \mathrm{O}_{2}(0.33 \\
\text { wt \%) }\end{array}$ & $\begin{array}{l}\text { Cleaning for } 10 \mathrm{~min} \\
\text { after each cycle }\end{array}$ & $\begin{array}{l}\text { Initial flux: } \\
\sim 280 \\
\left(\mathrm{~L} / \mathrm{m}^{2} \bullet \mathbf{h} \cdot \mathrm{bar}\right)\end{array}$ & $\sim 45 \%$ (after 3 cycles) \\
\hline $\begin{array}{l}\text { PVDF UF } \\
\text { [Desalination, 2015, } \\
369,51-61]\end{array}$ & $\begin{array}{l}\text { Seawater } \\
\text { including } \\
\text { dissolved } \\
\text { humic-based } \\
\text { organics and } \\
\text { salt (unclear) }\end{array}$ & $\begin{array}{l}\text { oxalic acid } \\
(\mathrm{OA}, 1 \%)+ \\
\mathrm{NaClO} \\
(1 \%)+ \\
\text { oxalic acid } \\
(1 \%)\end{array}$ & $\begin{array}{l}\text { Cleaning for } 30(\mathrm{OA})-60 \\
(\mathrm{NaClO})-30(\mathrm{OA}) \mathrm{min} \\
\text { after each cycle }\end{array}$ & $\begin{array}{l}\text { Initial } \\
\text { flux:142 LMH }\end{array}$ & $\begin{array}{l}25 \% \text { (after } 3 \text { cycles, } \\
\text { final flux: } 36.1 \mathrm{LMH} \text { ) }\end{array}$ \\
\hline $\begin{array}{l}\text { A composite PVDF } \\
\text { conductive } \\
\text { microfiltration (MF) } \\
\text { [Sci. Rep., 2015, 5, } \\
\text { 9268] }\end{array}$ & $\begin{array}{l}\text { Humic acid } \\
\text { (unclear) }\end{array}$ & $\begin{array}{l}\text { An electric } \\
\text { field of } 2 \\
\mathrm{~V} / \mathrm{cm}\end{array}$ & $\begin{array}{l}\text { Tests were conducted for } \\
\text { three cycles and each } \\
\text { lasted for } 3.0 \mathrm{~h}\end{array}$ & Not available & $\sim 50 \%$ (after 3 cycles) \\
\hline $\begin{array}{l}\text { Ceramic UF } \\
\text { membrane [J. } \\
\text { Membrane. Sci., } \\
\text { 2008, 311, 165-172] }\end{array}$ & $\begin{array}{l}\text { Lake Lansing } \\
\text { (Haslett, } \\
\text { Michigan), TOC } \\
(11.8 \mathrm{ppm})\end{array}$ & $\begin{array}{l}\mathrm{O}_{3}(9.5 \\
\left.\mathrm{g} / \mathrm{m}^{3}\right)\end{array}$ & gas flow rate of $0.2 \mathrm{~L} / \mathrm{min}$ & $\begin{array}{l}\text { Initial flux } \\
143 \\
\left(\mathrm{~L} / \mathrm{m}^{2} \bullet \mathrm{h} \cdot \text { bar }\right)\end{array}$ & $\begin{array}{l}\sim 82 \% \text { (after } 2 \mathrm{~L} \text { water } \\
\text { filtration) }\end{array}$ \\
\hline $\begin{array}{l}\text { FeOx-derived } \\
\text { ceramics membrane } \\
\text { [J. Membrane. Sci., } \\
\text { 2008, 311, 165-172] }\end{array}$ & $\begin{array}{l}\text { Humic acid (10 } \\
\text { ppm) }\end{array}$ & $\begin{array}{l}5 \mathrm{mM} \mathrm{H}_{2} \mathrm{O}_{2} \\
\text { treatment, } \\
\text { pH } 2.5(9 \\
\text { wt } \%)\end{array}$ & $\begin{array}{l}\text { After filtrated } 350 \mathrm{~mL} \mathrm{HA} \\
\text { feeding solution, cleaning } \\
\text { with } \mathrm{H}_{2} \mathrm{O}_{2}\end{array}$ & Not available & $\begin{array}{l}\sim 90 \% \text { (after } 600 \mathrm{~mL} \\
\text { water filtration) }\end{array}$ \\
\hline $\begin{array}{l}\text { Composite membrane } \\
\text { [J. Membrane. Sci., } \\
\text { 2004, 241, 143-160] }\end{array}$ & $\begin{array}{l}\text { Ground-water, } \\
\text { TOC (3.5-4.0 } \\
\text { ppm), UVA } 254 \\
(0.19-0.22)\end{array}$ & $\begin{array}{l}\mathrm{UV} / \mathrm{H}_{2} \mathrm{O}_{2} \\
\text { treated } \\
\text { water } \\
{\left[\mathrm{H}_{2} \mathrm{O}_{2}\right]=2} \\
\mathrm{mM}(3.6 \\
\mathrm{wt} \%)\end{array}$ & 45-min cleaning & $\begin{array}{l}\text { Initial flux } 11 \\
\left(\mathrm{~L} / \mathrm{m}^{2} \bullet \mathrm{h} \bullet \text { bar }\right)\end{array}$ & $\begin{array}{l}91 \% \text { (final flux } \sim 10 \\
\mathrm{~L} / \mathrm{m}^{2} \cdot \mathrm{h} \cdot \text { bar) }\end{array}$ \\
\hline $\begin{array}{l}\text { PVDF UF [J. } \\
\text { Membrane. Sci., } \\
\text { 2016, 505, 15-25] }\end{array}$ & $\begin{array}{l}\text { Humic acid } \\
(1000 \mathrm{ppm})\end{array}$ & $\begin{array}{l}\mathrm{O}_{3}(4.0 \\
\mathrm{mg} / \mathrm{L})\end{array}$ & $\begin{array}{l}1.0 \mathrm{mg} / \mathrm{min} \text {, } \\
\text { Ozonation reactions for } \\
30 \mathrm{~min}\end{array}$ & Not available & $\begin{array}{l}\sim 43 \% \text { (after } 1 \mathrm{~L} \\
\text { feeding solution } \\
\text { filtration) }\end{array}$ \\
\hline $\begin{array}{l}\text { PES UF [Chem. Eng. } \\
\text { J., 2018, 332, 76-84] }\end{array}$ & $\begin{array}{l}\text { Humic acid ( } 5 \\
\text { ppm) }\end{array}$ & $\begin{array}{l}\mathrm{NaClO}(200 \\
\text { ppm, } 250 \\
\mathrm{~mL})\end{array}$ & $\begin{array}{l}\mathrm{NaClO} \text { cleaning after } \\
\text { filtrated } 200 \mathrm{~mL} \text { feeding } \\
\text { solution }\end{array}$ & Not available & Around $80 \%-90 \%$ \\
\hline $\begin{array}{l}\text { PES UF [Water. Res, } \\
2001,35,3301-3308 \text { ] }\end{array}$ & $\begin{array}{l}\text { Orange County } \\
\text { groundwater, } \\
\text { humic content } \\
(\sim 6 \mathrm{ppm})\end{array}$ & $\begin{array}{l}\mathrm{NaOH}(0.1 \\
\mathrm{mol} / \mathrm{L})\end{array}$ & $\begin{array}{l}\text { Every continuous } 24 \mathrm{~h} \\
\text { filtration as a cycle, } \\
\text { soaked in } \mathrm{NaOH} \text { solution } \\
\text { for } 24 \mathrm{~h} \text { after each cycle. }\end{array}$ & $\begin{array}{l}\text { Initial flux } \\
(\sim 23 \mathrm{~mL} / \mathrm{min})\end{array}$ & $\begin{array}{l}30 \% \text { (end flux } \sim 7 \\
\mathrm{~mL} / \mathrm{min} \text { after three } \\
\text { cycles) }\end{array}$ \\
\hline
\end{tabular}

